\section{The missing link}

\author{
Viruses revise evolutionary theory
}

\section{Philip Hunter}

$\mathrm{V}$ iruses have gained a notoriously bad reputation as infectious agents, but they are nonetheless fascinating for biologists, not least because they are the most numerous and fastest evolving organisms on the planet. Moreover, viruses have had a significant role in the evolution of all other organisms, from bacteria through vertebrates to humans. But the full extent of this role is only just emerging, and scientists are reappraising not just the relationship between viruses and their hosts, but also the main principle of evolution: that it operates largely on the basis of gene mutation coupled with selection.

\section{...viruses have had a significant role in the evolution of all other organisms, from bacteria through vertebrates to humans}

In fact, infectivity is only the tip of the viral iceberg, and cohabitation is actually the norm-as is the case for bacteria. Similarly to bacteria and other microorganisms, most viruses and their hosts have co-evolved either into commensal relationships where the parasite's impact is neutral, or into mutually beneficial symbiotic arrangements. Of course, for viruses there is also the additional genomic dimension: they insert themselves into the host's DNA and in doing so can generate novel proteins and RNA molecules that might be valuable to the host. Moreover, it also allows the virus to manipulate the host genomic structure itself. Although this ability could wreak potential genetic havoc, it might also trigger significant structural and functional changes in the host genome that could not occur through random mutation alone.

$\mathrm{V}$ iruses have the unique ability to incorporate themselves permanently into the genomes of their hosts-a phenomenon known as endogenization-and thus lose their ability to infect other individuals. In return, they gain immortality, as they are passed down to successive generations of hosts.

Yet, although no other microorganisms can invade the genome, endogenization has a parallel among bacteria, which had just as profound a role in evolution. There is now widespread agreement that the mitochondria in eukaryotic cells probably originated as endogenous bacteria that were compromised and retained by the host. Their incorporation facilitated one of the biggest accelerations in evolution by giving eukaryotic life the ability to harness oxygen to convert glucose into a storable form of energy, ATP. Similarly, chloroplasts were endogenized bacteria that enabled their host cells to synthesize glucose by using light. Both mitochondria and chloroplasts retain ample evidence of their bacterial origins in the structure of their ribosomes and the circular arrangement of their chromosomes. However, mitochondria have shed most of their bacteria-like functions - retaining only those required by the host cell-and have transferred some of their genes to the nucleus.

Likewise, endogenous viruses have lost their pathogenic abilities but have retained viral-like DNA structures, such as long terminal repeat sequences, which RNA viruses use to insert their genes into the host genome. Similarly to the bacterial ancestors of mitochondria and chloroplasts, endogenous retroviruses have introduced fundamental functions, such as the ability to form syncytia: cellular conglomerates that contain multiple nuclei.

Syncytia underpin several crucial structural and functional roles in vertebrates, especially mammals. In vertebrates, skeletal muscles develop through the fusion of myocite cells into long fibres. Similarly, fibre cells fuse to form the lens of the eye, and macrophage/monocyte-derived cells fuse to form large phagocytes involved in immune surveillance and bone resorption, which has a key role in the ageing process. Intriguingly syncytin, the protein encoded by an endogenous retrovirus that induces the formation of syncytia, is expressed densely in the mammalian brain, suggesting it has an important, as-yet-undiscovered role there. One explanation is that syncytin might be a component in the repair of damaged neurons, by enabling the existing cellular fragments to fuse together (Mohler, 2006).

A Ithough endogenous retroviruses have not yet been associated directly with most of these processes, they have been shown to provide a crucial function during placenta formation in mammals. The placenta's key component is a membrane through which nutrients are transferred from mother to fetus and waste products are removed. Recent studies have demonstrated that placenta formation is impossible without the presence of a gene coding for an envelope protein from an endogenous retrovirus, which has different names depending on the host mammal: HERV-W in humans and enJSRV in sheep. "We have shown that there is no successful pregnancy in sheep without enJSRV envelope expression," explained Massimo Palmarini from the University of Glasgow in the UK (Dunlap et al, 2006). "Similar results have recently been shown in mice and have been hypothesized in humans by experiments in vitro" (Malassiné et al, 2008).

\section{...infectivity is only the tip of the viral iceberg, and cohabitation is actually the norm...}

Many viruses have membrane envelopes made largely of proteins, sometimes combined with lipids or oligosaccharides, which comprise a bag of tricks necessary for invading a host cell. Human immunodeficiency virus (HIV), for example, expresses a glycoprotein called gp120 that is anchored to the viral envelope, which binds to the CD4 receptor on several immune cells. On binding, the virus fuses its membrane with that of the host cell as part of the integration process. After the viral genes have integrated into the host genome, the cell produces HIV envelope proteins that migrate back to the host cell membrane, from which the HIV gp120 protein then protrudes. In this way, the HIV-virus-infected cells resemble virus particles externally. This causes infected T cells to fuse with non-infected $\mathrm{T}$ cells through the same gp120/CD4 binding process that the virus 


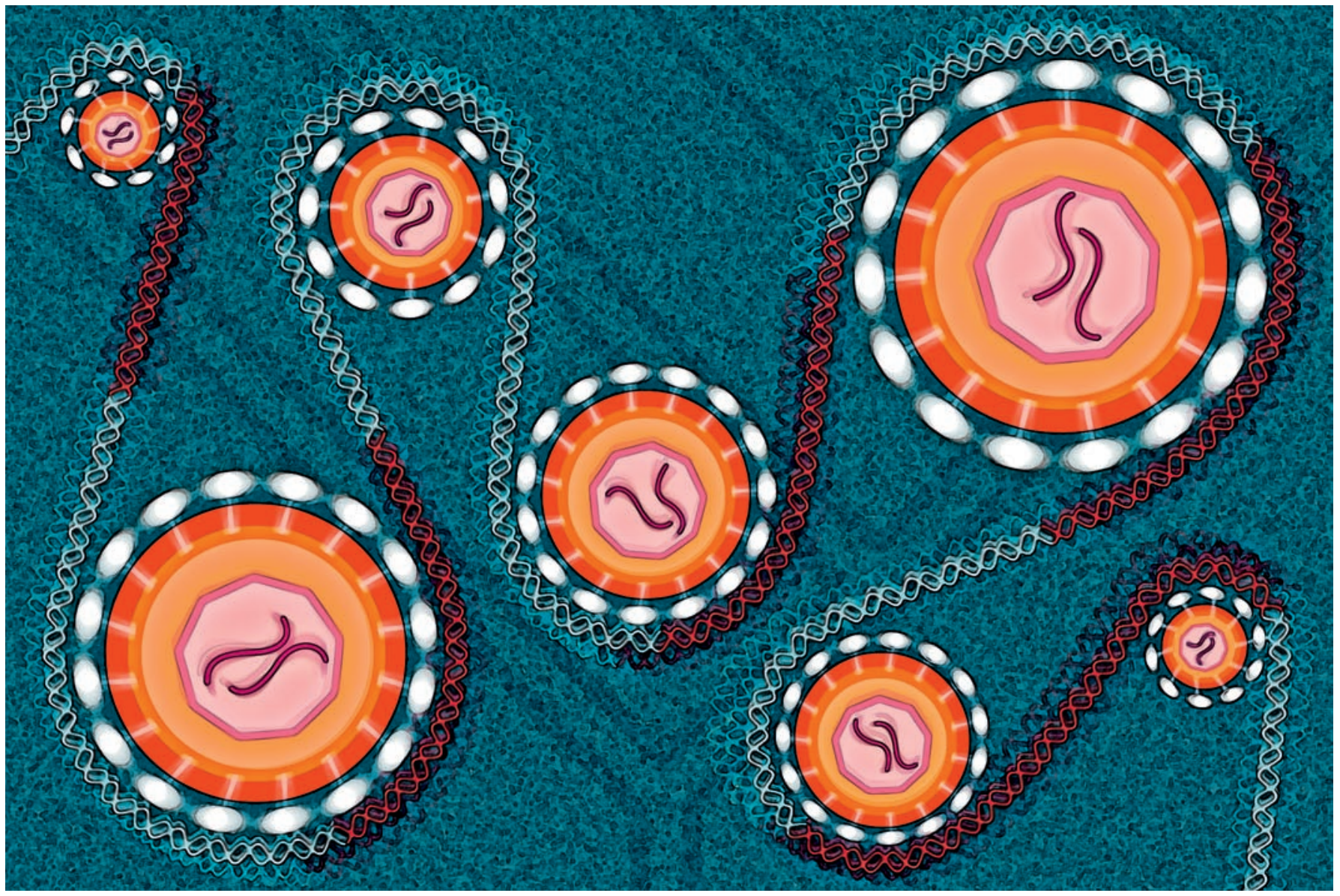

used for the initial invasion. Eventually, several healthy $\mathrm{T}$ cells fuse together with an infected T cell to create a single large HIVinfected cell with up to 500 nuclei. This cell fusion process is involved in one of the most devastating diseases of recent times, and yet has also been recruited for crucial roles in vertebrate and mammalian evolution.

It seems that non-viral vertebrate gene products cannot create syncytia, although they are an integral part of the viral machinery needed to enter a cell. The role of syncytia has led some virologists, such as Frank Ryan from Sheffield University in the UK, to hypothesize that viruses have had a major role in the evolution of higher organisms by enabling the latter to vault evolutionary chasms that perhaps would be insurmountable by stepwise mutation.

According to Ryan, viruses are the "missing link" in evolution, operating in symbiotic union with the host and providing a source of functional novelty and gene transcription control. In his recent book Virolution, Ryan described a number of endogenous retrovirus sequences in
The role of syncytia has led some virologists [...] to hypothesize that viruses have had a major role in the evolution of higher organisms by enabling the latter to vault evolutionary chasms...

humans that are responsible for important functions or tissues, including BRCA 1 , the keratin gene required for skin and eye structure, the gene responsible for the starchdigesting enzyme amylase, and various genes involved in immunity (Ryan, 2009). Such retroviral sequences are also implicated in genes that have important roles in some cancers, including breast cancer and the so-called Wilms' tumour that affects the kidneys of some children.

Ryan also pointed out that, in the placenta, the role of endogenous viruses is not confined to the membrane structure. "We now know of eight viruses that play an important role in placental function, with roles much wider than just syncytium formation," he explained. "This is clearly of major importance, but these additional roles haven't all been worked out as yet."

I he main question of how viruses are recruited as permanent allies within the host genome, or how they become 'endogenized', remains unanswered. Ryan has developed a novel theory known as 'plague culling', which proposes that aggressive invasion by some retroviruses is a prelude to the subsequent establishment of a commensal or even mutualistic symbiotic relationship with the surviving members of the host species that have become immune to the virus. Ryan argues that this has happened with increasing frequency during mammalian and primate evolution, and even that the current AIDS epidemic is part of that process: HIV itself could in time be integrated into our genomes and gain a crucial role.

For endogenization to occur, the viral genome must unite directly with the host genome at the germ-line level, which is an ability possessed by retroviruses. As Ryan pointed out, eukaryotic genomes contain 
a large number of genetic elements called long interspersed nuclear elements (LINEs) and short interspersed nuclear elements (SINEs), which he describes as decayed products of past symbiotic endogenous retroviruses. He draws an analogy with eukaryotic mitochondria, whose genome has been stripped down during two billion years of evolution. Similarly, LINEs and SINEs are truncated versions of the original virus, but retain some of its behavioural, structural and functional properties.

Not all retroviruses unite with their hosts at the genome level; instead, they coexist in an exogenous rather than endogenous relationship. Similarly to DNA viruses, exogenous retroviruses combine with their host at the tissue rather than germ-line level and exploit the cellular machinery, such as RNA transcriptase and ribosomes, to express their genes. In doing so, they retain their independent status and ability to cause infection.

\section{...HIV itself could in time be integrated into our genomes and gain a crucial role}

In this guise, exogenous retroviruses can also enter stable mutualistic symbiotic relationships with their hosts after plague culling has taken place, according to Luis Villarreal-a Mexican virologist now at the University of California in Irvine, USA, whose research has ranged widely over the role of viruses in vertebrate adaptive immunity. In this way, the virus gains a semipermanent residence, free from attack by the host's immune system. In turn, the host gains a potent competitive weapon against closely related species that have not been exposed to it and therefore lack immunity.

I n fact, plague culling is not confined to retroviruses but is shared by many DNA viruses, a number of which might persist harmlessly in mammalian populations, spread perhaps from mother to offspring during lactation. When introduced to a related species or population, the virus becomes lethal again, instigating another round of plague culling. This can happen as a result of migration, or recently in the case of some mammals, through deliberate or accidental human intervention: "The current dying off of the red squirrels in England due to invasion by the grey squirrel is due to the persisting squirrel pox of the grey squirrel that is lethal to the red squirrel," noted Villarreal.

But here too are signs that the relationship between the red squirrel and the pox virus might be entering a second phase of cohabitation, with the emergence of resistant individuals in parts of England (Sainsbury et al, 2008). Whether viruses acting in this way undergo active selection is uncertain, but this property of remaining lethal to other species certainly enhances the fitness of both parties. For the virus it provides a mechanism of extending its population when its host encounters closely related groups; for the host it helps repel invaders, and according to Villarreal, might have contributed to the extinction of the Neanderthals through contact with modern humans. "We can even think of the recent European colonization of the New World as a process that was significantly aided by the group-specific action of viruses to which the Europeans were adapted at a population level as childhood infections," he said.

Villarreal commented that viruses definitely have a role in relationships between humans and primates too, and cross infection can prove lethal in either direction. "For example, I suggest you never let a caged primate spit in your eye, because [the] herpes $\mathrm{B}$ virus prevalent in many monkeys is lethal for about $50 \%$ of humans. Nor should you enter a Gibbon colony with an open herpes lip sore as this virus can wipe out the colony."

A part from this putative role in competition between related groups and species, viruses-both exogenous and endogenous-have had more obvious roles in evolution, even to the point of forming obligate mutualistic symbioses. Parasitic wasps are one of the most studied examples; most of the species have evolved an obligate symbiosis with polydnaviruses, which subdue the immune system of the host caterpillars in which they lay their eggs. Jean-Michel Drezen and colleagues at the University François Rabelais in Tours, France, have recently identified some of the genes that prevent the host immune system from attacking the eggs of the wasp (Bézier et al, 2009). The relationship between virus and wasp has proven to be highly successful and has been adopted by at least 10,000 species of parasitic wasp; moreover, it has evolved to minimize damage to the caterpillars, whose fitness, after all, is important for both wasp and virus. Production of the polydnavirus particles is confined to specialized cells in the wasp ovaries and does not occur inside the caterpillars. The virus has also been stripped of structural proteins, leaving just the products necessary for manipulating the physiology of the caterpillars to allow the wasp eggs to grow.

\section{...accumulating evidence from viral research indicates that Darwin's theory of evolution cannot alone explain all aspects of life's development...}

There are also some recently studied examples of obligate mutualistic relationships involving plants and viruses. In some cases, the virus seems to act as a facilitator between the plant and some other parasite, as in the mutualistic symbiosis between a fungal endophyte and a tropical panic grass, which enables both to withstand heat. Research by Marilyn Roosinck and colleagues at the Samuel Roberts Noble Foundation in Ardmore (OK, USA) has found that this apparently two-organism mutualistic symbiosis actually works only in the presence of a third partner: the Curvularia thermal tolerance virus that infects the fungus (Márquez et al, 2007). Fungal isolates cured of the virus failed to confer heat tolerance, but were able to do so upon reinfection with the virus. Furthermore, the virus-infected fungus confers heat tolerance not only to its native host, but also to different plant species, which suggests that the underlying mechanism-which is still unknown-has been conserved in the plant kingdom.

Roosinck, like Ryan and Villarreal, believes that accumulating evidence from viral research indicates that Darwin's theory of evolution cannot alone explain all aspects of life's development, but that the gaps can be filled by combining it with the symbiotic theory of evolution. In addition to point mutation in a species, phenotypic novelty would also occur through recombination between different species, leading to genomic reassortment. The idea that this has happened among viruses is no longer controversial. However, the idea that recombination between viruses and their hosts has had a significant role in the evolution of the latter, as proposed by Ryan and others, is much more controversial and 
will provide a focus for research in both evolutionary biology and medicine in years to come.

\section{REFERENCES}

Bézier A, Herbinière J, Lanzrein B, Drezen JM (2009) Polydnavirus hidden face: the genes producing virus particles of parasitic wasps. J Invertebr Pathol 101: 194-203

Dunlap KA, Palmarini M, Varela M, Burghardt RC, Hayashi K, Farmer JL, Spencer TE (2006) Endogenous retroviruses regulate periimplantation placental growth and differentiation. Proc Natl Acad Sci USA 103: 14390-14395

Malassiné A, Frendo JL, Blaise S, Handschuh K, Gerbaud P, Tsatsaris V, Heidmann T,

Evain-Brion D (2008) Human endogenous retrovirus-FRD envelope protein (syncytin 2) expression in normal and trisomy 21 -affected placenta. Retrovirology 5: 6

Márquez LM, Redman RS, Rodriguez RJ,

Roossinck MJ (2007) A virus in a fungus in a plant: three-way symbiosis required for thermal tolerance. Science 315: 513-515
Mohler WA (2006) Transient Channels Leading to Plasma Membrane Merger. In Cell-Cell Fusion, pp298-316. Heidelberg, Germany: Springer Ryan F (2009) Virolution. London, UK: Collins Sainsbury AW et al (2008) Poxviral disease in red squirrels Sciurus vulgaris in the UK: spatial and temporal trends of an emerging threat. Ecohealth 5: 305-316

\section{Philip Hunter is a freelance journalist} in London, UK.

EMBO reports (2010) 11, 28-31.

doi:10.1038/embor.2009.267

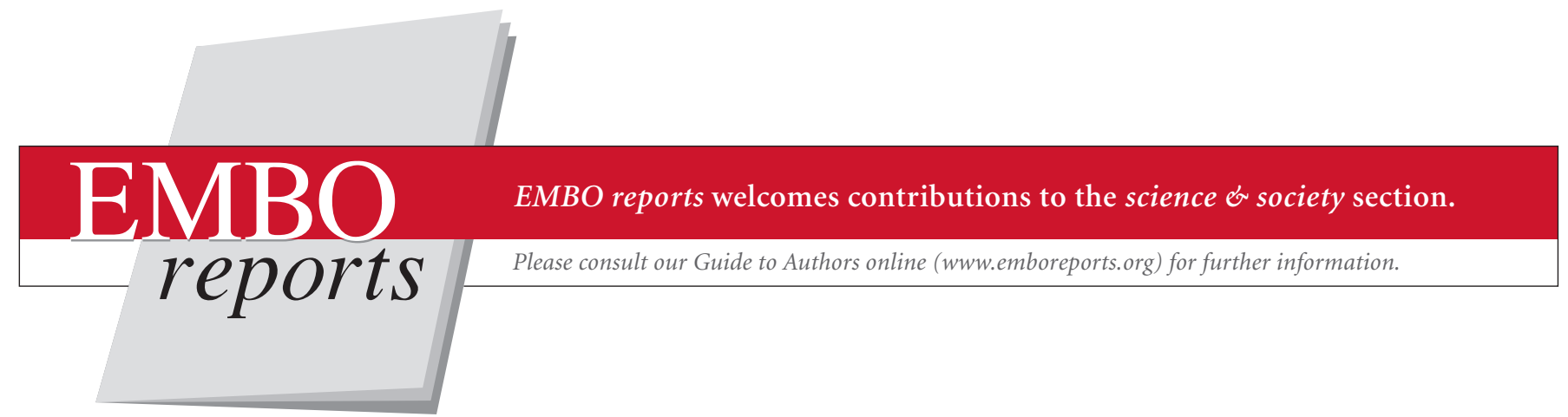

